\title{
PNEUMOTÓRAX ESPONTÂNEO SECUNDÁRIO A DOENÇA PULMONAR OBSTRUTIVA CRÔNICA EM FELINO
}

\author{
Manuela Bamberg Andrade ${ }^{1,}$ Adriane Pimenta da Costa Val Bicalho ${ }^{2}$
}

${ }^{1}$ Aluna de Pós-graduação em Ciência Animal, Escola de Veterinária da UFMG, Universidade Federal de Minas Gerais ${ }^{2}$ Prof $^{\text {a }}{ }^{-2} r^{\text {a }}$ Departamento de Clinica e Cirurgia

Veterinárias, Universidade Federal de Minas Gerais (adriane@ufmg.br)

Recebido em: 02/10/2017 - Aprovado em: 21/11/2017 - Publicado em: 05/12/2017

DOI: 10.18677/EnciBio_2017B48

\begin{abstract}
O pneumotórax é a presença de gás no espaço pleural, podendo ser de origem traumática ou espontânea. O pneumotórax espontâneo primário ocorre quando não se observa causa traumática ou moléstia pulmonar subjacente e já no pneumotórax espontâneo secundário observam-se doenças de vias aéreas ou pulmonares primárias, que levam à hiperinsuflação do pulmão. Admite-se que a causa do pneumotórax espontâneo primário seja a ruptura de bolha ou cisto subpleurais. Não há relatos de pneumotórax espontâneo primário em felinos e dos poucos sobre pneumotórax espontâneo secundário, nenhum tem como afecção de base a doença pulmonar obstrutiva crônica. Os sinais clínicos do pneumotórax são agudos, cursando com dispneia, taquipneia e cianose. O tratamento pode ser cirúrgico e corresponde inicialmente no alívio da dispneia por toracocentese ou adaptação de dreno torácico. O prognóstico do pneumotórax espontâneo tende a ser reservado a desfavorável. Relata-se o primeiro caso de pneumotórax espontâneo em felino gerado por doença pulmonar obstrutiva crônica.
\end{abstract}

RESUMO

PALAVRAS-CHAVE: Bronquite Crônica, Dispneia, Felinos Pulmão.

\section{SPONTANEOUS PNEUMOTHORAX SECONDARY TO CHRONIC OBSTRUCTIVE PULMONARY DISEASE IN FELINE}

\begin{abstract}
Pneumothorax is the presence of gas in the pleural space, and may be of traumatic or spontaneous origin. Primary spontaneous pneumothorax occurs when there is no traumatic cause or underlying lung disease, and in the secondary spontaneous pneumothorax, primary airway or pulmonary diseases, which lead to hyperinflation of the lung, are observed. It is assumed that the cause of primary spontaneous pneumothorax is a subpleural blister or cyst rupture. There are no reports of primary spontaneous pneumothorax in felines and of the few of secondary spontaneous pneumothorax, none of them is based on chronic obstructive pulmonary disease. The clinical signs of pneumothorax are acute, leading to dyspnea, tachypnea and cyanosis. The treatment may be surgical and initially corresponds to the relief of the ENCICLOPÉDIA BIOSFERA, Centro Científico Conhecer - Goiânia, v.14 n.26; p.508 2017
\end{abstract}


dyspnea by thoracentesis or continuous thoracic drainage. Prognosis of spontaneous pneumothorax tends to be restricted to unfavorable. We report the first case of a cat with spontaneous pneumothorax due to chronic obstructive pulmonary disease.

KEYWORDS: chronic bronchitis, dyspnea, feline, lung.

\section{INTRODUÇÃO}

O pneumotórax é a presença de gás no espaço pleural e pode ser de origem traumática ou espontânea, sendo que este ocorre sem um evento traumático conhecido e pode ser classificado como primário ou secundário (MANCHI et al., 2017) e são bem documentados em humanos e cães.

O pneumotórax espontâneo primário ocorre como resultado de rompimento de bolhas pulmonares, não está associado à doença pulmonar adjacente e nunca foi relatado em felinos. Encontram-se poucos relatos de pneumotórax espontâneo secundário nesta espécie, que estão associados principalmente à asma (MOONEY et al., 2012, LIU; SILVERSTEIN, 2014), pneumonia (MOONEY et al., 2012) neoplasias (GRECl et al., 2015) (MOONEY et al., 2012) e abscessos pulmonares. Os sinais clínicos principais são dispneia e cianose e abordagem emergencial se faz por toracocentese (RABELO; CROWE, 2005).

A lobectomia é o tratamento para resolução mais rápida de sinais clínicos, e o prognóstico do pneumotórax espontâneo é de reservado à desfavorável (LIU; SILVERSTEIN, 2014). Este trabalho relata um caso de pneumotórax espontâneo secundário à doença pulmonar obstrutiva crônica em felinos, o primeiro descrito na literatura.

\section{RELATO DE CASO}

Atendeu-se no Hospital Veterinário da UFMG, um felino sem raça definida, fêmea, de aproximadamente 13 anos. O tutor relatou que o animal apresentava há aproximadamente três meses quadro de tosse que se agravava progressivamente e que havia sido medicada anteriormente com anti-inflamatório esteroidal (dexametasona) e, cefoveccina sódica com melhora pouco significativa e efêmera.

$\mathrm{Na}$ anamnese relatou-se apatia, hiporexia, fezes ressecadas, normoúria e normodipsia. Ao exame clínico geral o animal mostrava estado nutricional ruim, mucosas oculares normocoradas, tempo de preenchimento capilar não avaliado, por hiperpigmentação da mucosa oral, linfonodos normorreativos e desidratação estimada em 6\%, frequência cardíaca de 196 bpm, frequência respiratória de 160 movimentos por minuto e temperatura retal de $37,1^{\circ} \mathrm{C}$.

Ao exame clínico do sistema respiratório o animal apresentava taquipneia com dispneia expiratória grave; hipofonese de bulhas cardíacas e à ausculta pulmonar revelou roçar de pleuras. O animal foi admitido na Unidade de Terapia Intensiva com as suspeitas iniciais de pneumotórax, efusão torácica e neoplasia pulmonar e logo submetido à toracocentese segundo a técnica descrita por Rabelo e Crowe (2005) pois seu estado clinico não permitia exames de imagem preliminares. Houve drenagem de grande volume de ar. Não foram observadas alterações significativas no hemograma.

Entretanto, a análise bioquímica revelou aumento de alanina aminotransferase (ALT) para 201, $69 \mathrm{U} / \mathrm{L}$ (valor de referência 6-83 U/L) bem como de aspartato aminotransferase (AST) para 190,31 U/L (valor de referência 2343U/L). O teste rápido de FIV/FeLV apresentou resultado negativo. Após a estabilização da paciente, realizou-se radiografia de tórax e observou-se ainda 
grande quantidade de pneumotórax bilateral. Não havia sinal de neoplasia nem evidência de trauma. O pulmão apresentava apenas padrão bronquial. $O$ animal foi medicado inicialmente após a toracocentese com anti-inflamatório (meloxicam $0,05 \mathrm{mg} / \mathrm{kg}$ ), analgésico (tramadol $2,0 \mathrm{mg} / \mathrm{kg}$ ), inibidores da bomba de prótons (omeprazol $1 \mathrm{mg} / \mathrm{kg}$ ) e anti-emético (ondansetrona $1 \mathrm{mg} / \mathrm{kg}$ ).

Após três dias de internação a formação de pneumotórax perpetuou-se, como evidenciaram radiografias torácicas. Decidiu-se a colocação cirúrgica de dreno torácico (FRENDIN; OBEL, 1997), do lado esquerdo, que drenava maior quantidade de ar nas toracocenteses. A aspiração foi realizada continuamente, a cada três horas, até que se alcançasse a melhora clínica da paciente e manutenção da pressão intratorácica negativa (RABELO; CROWE, 2005). A alimentação foi fornecida por sonda esofágica (GAJANAYAKE, 2014). Para esses procedimentos o animal foi acondicionado em ventilação mecânica. Após melhora significativa do quadro respiratório, o animal foi retirado da ventilação mecânica.

Todavia, no quinto dia de internação o animal apresentou respiração agônica, mesmo com drenagem contínua de grande volume de ar. Decidiu-se então a realização de toracotomia exploratória. A cavidade torácica foi inspecionada e identificou-se que o pulmão esquerdo estava totalmente atelectasiado, enquanto que pulmão direito apresentava lesões puntiformes amareladas, mas em seu restante aspecto, consistência e coloração aparentemente sem alteração. Quando o pulmão direito foi insuflado todos os lobos inflavam sem aparente alteração. A partir deste achado decidiu-se realizar a toracotomia intercostal para obter melhor acesso ao pulmão esquerdo, em seguida pneumectomia esquerda como indicado por Majeski et al., (2016).

A cavidade torácica foi preenchida com solução fisiológica aquecida, o pulmão direito foi insuflado e verificou-se a ausência de extravasamento de ar, bem como do coto do brônquio principal esquerdo. O líquido foi removido com o uso de um aspirador cirúrgico (FRENDIN; OBEL, 1997). O animal se manteve estável durante a anestesia, sendo possível a retirada da ventilação mecânica e a retirada do traqueotubo, apresentando respiração espontânea. Todavia, logo em seguida a paciente apresentou parada cardiorrespiratória sem sucesso nas tentativas de reanimação.

O corpo foi encaminhado à necropsia. Ao exame macroscópico o pulmão esquerdo estava acentuadamente reduzido de tamanho, colapsado, firme e acrepitante. Os lobos cranial e caudal do pulmão direito apresentavam áreas esbranquiçadas, multifocais e firmes com material fibrilar brancacento na superfície. À microscopia o pulmão direito apresentava bronquite e bronquiolite, fibrose intraluminal bronquial multifocal acentuada com bronquiectasia, broncoestenose, hiperplasia da musculatura lisa e atelectasia difusa. O laudo histopatológico informou que o animal possuía uma doença pulmonar obstrutiva crônica.

\section{RESULTADOS E DISCUSSÃO}

De acordo com a anamnese, o animal apresentava tosse há pelo menos três meses, Segundo Nelson e Couto (2006), um dos sinais clínicos de um paciente com pneumotórax é a taquipneia e a ausculta pulmonar e cardíaca abafadas, o que foi evidenciado no exame clínico. A paciente apresentava pneumotórax sem nenhum histórico de trauma o que sugere pneumotórax espontâneo (GARBER et al., 2015), que poderia ser classificado como secundário, sugerindo aparente anormalidade 
estrutural ou funcional pulmonar (MANCHI et al., 2017).

O aumento de enzimas hepáticas pode estar relacionado com 0 desenvolvimento de lipidose hepática, que é o acumulo de gordura no fígado. Essa síndrome aparece em gatos que diminuem o consumo de alimento ou param de se alimentar por algum estresse. Dessa forma, há uma restrição de proteínas e carboidratos e a lipólise fica sendo um meio de obtenção de energia, acumulando assim, gordura no fígado, em mais de $80 \%$ dos hepatócitos (VALTOLINA; FAVIER, 2017). As anormalidades mais consistentes são encontradas no perfil bioquímico. $O$ nível sérico das enzimas alanina amino transferase (ALT) e aspartato amino transferase (AST) está quase sempre aumentado (VALTOLINA; FAVIER, 2017). Realizou-se toracocentese de emergência visto que a paciente se mostrava em angústia respiratória. A toracocentese evidenciou que se tratava de pneumotórax.

De acordo com Mooney et al., (2012), pacientes com instabilidade costal devem primeiro ser tratados quanto à condição cardiovascular e drenagem da cavidade torácica. Como a paciente não apresentava melhora do quadro e o pneumotórax se formava constantemente, optou-se por realizar toracotomia e isso se confirma por Rabelo e Crowe, (2005): "o paciente que tenha extravasamento contínuo de ar na cavidade torácica deve ser reavaliado e indica-se a toracotomia para correção do problema".

Durante a cirurgia constatou-se colapso total do pulmão esquerdo, sem que tivesse nenhuma função vital. Dessa forma, a retirada foi a melhor opção. Segundo Wavreille et al.,(2016), o lado afetado não impede a decisão de realizar a pneumonectomia quando o procedimento é indicado. Todavia, mesmo com todas as intervenções cabíveis, o animal não resistiu e veio a óbito. Entretanto, a toracotomia cirúrgica e a exploração do tórax em cães com pneumotórax espontâneo, apresenta elevada taxa de cura pós-operatória e morbidade pós-operatória limitada (MOONEY et al., 2012).

Tal desfecho em gatos reflete a gravidade e a natureza difusa da doença subjacente nestes gatos, em vez de uma complicação direta da toracotomia (MOONEY et al., 2012). O indivíduo pode viver apenas com um pulmão, porém desde que este esteja saudável. De acordo com Wavreille et al., (2016), existem provas documentadas de compensação após pneumonectomia que exceda $50 \%$ do volume pulmonar. Isso tem sido explicado por recrutamento de reservas fisiológicas e remodelagem da rede alveolar-capilar existente. A indicação primária de pneumonectomia em humanos é para tratamento de câncer pulmonar. Nos 14 casos de pneumonectomia até agora relatados na literatura veterinária, sete se deviam à neoplasia (WAVREILLE et al., 2016).

Segundo o laudo histopatológico, o pulmão direito apresentava quadro de doença pulmonar obstrutiva crônica; hiperplasia de musculatura lisa e fibrose intraluminal bronquial multifocal acentuada com bronquiectasia e broncoestenose, associado à hiperplasia de Tecido Linfoide Associado aos Brônquios (BALT) e à atelectasia multifocal a coalescente acentuada. Uma das principais doenças brônquicas dos felinos que se enquadram na doença pulmonar obstrutiva crônica é a bronquite crônica. Segundo Nelson e Couto, (2006), a bronquite crônica apresenta características como inflamação crônica das vias aéreas resultando em lesão irreversível como fibrose. Ainda segundo os autores a bronquite pode se desenvolver em gatos de qualquer idade.

Asma e bronquite crônica são doenças comuns em gatos e estão associadas à inflamação crônica das vias aéreas inferiores (SCHULZ et al., 2014). Todavia, há 
dificuldade na diferenciação entre as duas doenças, visto que os sinais clínicos são muito similares, além do que os métodos diagnósticos, como radiografia torácica e lavado traqueo-brônquico apresentam resultados semelhantes. O tratamento para ambas as enfermidades também é bastante similar. Estudos retrospectivos em gatos com doença das vias aéreas inferiores (asma e bronquite crônica) documentaram resposta clínica a glucocorticóides orais ou parenterais e / ou broncodilatadores (TRZIL; REINERO, 2014).

Existem vários termos para designar doenças brônquicas em felinos, dentre estes tem-se: asma brônquica, bronquite alérgica, bronquite crônica, bronquite asmática crônica, doença pulmonar obstrutiva felina (TRZIL; REINERO, 2014). Observa-se a real dificuldade na definição das doenças brônquicas dos felinos. Dessa forma, torna-se importante uma anamnese e exame clínico mais detalhado quanto ao sistema respiratório dos felinos, pois uma simples bronquite crônica quando não tratada ou quando tratada inadequadamente pode evoluir para um quadro mais grave, como o pneumotórax espontâneo secundário.

\section{CONCLUSÃO}

Embora o relato do tutor sugerisse apenas uma doença respiratória rotineira, os sinais clínicos eram compatíveis com pneumotórax ou derrame pleural. A assistência imediata ao animal permitiu que outros procedimentos tanto de diagnóstico quanto de tratamento fossem instituídos. Os achados laboratoriais foram consonantes com lipidose hepática, que acomete gatos hiporéxicos ou anoréxicos, como no caso em pauta. Apesar da intervenção cirúrgica adequada ao caso, a pneumoectomia, tenha sido instituída, o prognóstico desfavorável da afecção confirmou-se, devido ao grau de acometimento pulmonar.

No relato em questão, a causa do pneumotórax foi a doença pulmonar obstrutiva crônica. Até o momento, não houve descrição na literatura sobre essa enfermidade como causa de pneumotórax em felino.

Cabe aos veterinários terem especial atenção à DPOC em felinos, pois o diagnóstico e a instituição de tratamentos precoces podem evitar agravos pulmonares futuros, entre estes o pneumotórax.

\section{REFERÊNCIAS}

FRENDIN, J.; OBEL, N. Catheter drainage of pleural fluid collections and pneumothorax. Journal of Small Animal Practice, v. 38, n. 6, p. 237-242, jun. 1997. Disponivel em: < http://doi.wiley.com/10.1111/j.1748-5827.1997.tb03355.x>. doi: 10.1111/j.1748-5827.1997.tb03355.x

GAJANAYAKE, I. Management of the anorexic cat. In Practice, v. 36, n. 4, p. 163171, 2014. Disponivel em:

http://inpractice.bmj.com/lookup/doi/10.1136/inp.g2378>. doi: 10.1136/inp.g2378

GARBER, J. B.; SALE, K.; RADEMACHER, N.; WIGNALL, J. R.; MCLAUGHLIN, L. D.; RYAN, K. A. Pneumothorax in a dog caused by necrotizing pneumonia secondary to heatstroke. Journal of veterinary emergency and critical care (San Antonio, Tex. $\square:$ 2001), v. 25, n. 6, p. 759-764, nov. 2015. Disponivel em: < http://doi.wiley.com/10.1111/vec.12361 >. doi: 10.1111/vec.12361

GRECI, V.; BAIO, A.; BIBBIANI, L.; CAGGIANO, E.; BORGONOVO, S.; et al. 
Pneumopericardium,

pneumomediastinum,

pneumothorax

and

pneumoretroperitoneum complicating pulmonary metastatic carcinoma in a cat. Journal of Small Animal Practice, v. 56, n. 11, p. 679-683, 2015. Disponivel em: < http://doi.wiley.com/10.1111/jsap.12366>. doi: 10.1111/jsap.12366

LIU, D. T.; SILVERSTEIN, D. C. Feline secondary spontaneous pneumothorax: A retrospective study of 16 cases (2000-2012). Journal of Veterinary Emergency and Critical Care, v. 24, n. 3, p. 316-325, 2014. Disponivel em: < http://doi.wiley.com/10.1111/vec.12150>. doi: 10.1111/vec.12150

MAJESKI, S. A.; STEFFEY, M. A.; MAYHEW, P. D.; HUNT, G. B., HOLT, D. E.; JEFFREY, J., et al. Postoperative Respiratory Function and Survival After Pneumonectomy in Dogs and Cats. Veterinary Surgery, v. 45, n. 6, p. 775-781, 2016. Disponivel em: <http://doi.wiley.com/10.1111/vsu.12513>. doi: 10.1111/vsu.12513

MANCHI, G.; JAROLMASJED, S.; BRUNNBERG, M.; SHAHID, M.; REHBEIN, S.; et al. Spontaneous pneumothorax in cats $\square$ : two case reports and literature review. Tierärztliche Praxis Ausgabe K: Kleintiere / Heimtiere n. 16, p. 1-7, 2017. Disponivel em: < http://www.schattauer.de/index.php?id=1214\&doi=10.15654/TPK150466>. doi: 10.15654/TPK-150466

MOONEY, E. T.; ROZANSKI, E. A.; KING, R. GP.; SHARP, C. R; Spontaneous pneumothorax in 35 cats (2001-2010). Journal of Feline Medicine and Surgery, v. 14, n. 6, p. 384-391, 2012. Disponivel em: <http://journals.sagepub.com/doi/10.1177/1098612X12439947>.

10.1177/1098612X12439947

NELSON, R.W.; COUTO, C.G. Medicina Interna de Pequenos Animais. 3.ed. Rio de Janeiro: elsevier, p. $280-286,2006$.

RABELO, R. C., CROWE, D. T. Fundamentos de Terapia Intensiva Veterinária em Pequenos Animais - condutas no paciente crítico. 1 ed, Rio de Janeiro: I. f. livros de veterinária Itda, 2005.

SCHULZ, B. S.; RICHTER, P.; WEBER, K.; MUELLER, R. S.; WESS, G. et al. Detection of feline Mycoplasma species in cats with feline asthma and chronic bronchitis. Journal of Feline Medicine and Surgery, v. 16, n. 12, p. 943-9, 2014. Disponivel em: <http://journals.sagepub.com/doi/10.1177/1098612X14524969>.doi: $10.1177 / 1098612 \times 14524969$

TRZIL, J. E.; REINERO, C. R. Update on feline asthma. Veterinary Clinics of North America - Small Animal Practice, v. 44, n. 1, p. 95-105, 2014. Disponivel em: <http://linkinghub.elsevier.com/retrieve/pii/S0195561613001794>. doi: 10.1016/j.cvsm.2013.08.006

VALTOLINA, C.; FAVIER, R. P. Feline Hepatic Lipidosis. Veterinary Clinics of North America - Small Animal Practice, v. 47, n. 3, p. 683-702, 2017. Disponivel em: $\quad$ <http://linkinghub.elsevier.com/retrieve/pii/S0195561616301607>.doi: 10.1016/j.cvsm.2016.11.014

WAVREILLE, V.; BOSTON, S. E; SOUZA, C.; HAM, K.; CHANOIT, G. et al. Outcome After Pneumonectomy in 17 Dogs and 10 Cats: A Veterinary Society of Surgical 
Oncology Case Series. Veterinary Surgery, v. 45, n. 6, p. 782-789, 2016. Disponivel em: <http://doi. wiley.com/10.1111/vsu.12517>.doi: 10.1111/vsu.12517 